\title{
Investigation on Seasonality of Twin Births in Brazil
}

\author{
H. Krieger ${ }^{1}$, G. M. D. D. Colletto', C. Franchi-Pinto ${ }^{2}$, B. Beiguelman² \\ 'Department of Parasitology, University of São Paulo, S. Paulo, SP, Brazil; ${ }^{2}$ Depart- \\ ment of Medical Genetics, School of Medicine, UNICAMP, Campinas, SP, Brazil
}

\begin{abstract}
The hypothesis of seasonality of twin births was investigated in two important maternity hospitals in the State of São Paulo, Brazil. The study included 1,386 twin births that occurred among 154,699 deliveries from 1984 to 1993. No evidence of seasonality has been detected either for the twin birth rate considered as a whole or for dizygotic twinning rate. The distributions of these rates fitted well sinusoidal regression curves but the cyclic trend did not correspond to any specific season.
\end{abstract}

Key words: Seasonality, Periodicity, Cyclic variation, Twin births, Dizygotic rate, Twin births in southeastern Brazil

\section{INTRODUCTION}

Reports on seasonal variation in twin birth rates are conflicting, since some authors found no evidence of seasonality in twinning [7,20,23] while others, who suggested that such variation exist, found no coincident results $[1,3-6,8-19,21]$. Moreover, in the later reporters it is not always clear whether the conclusion favoring seasonality of twin births was based on the recognition of a cyclic trend or merely by the observation of a seasonal excess in a monthly distribution of pooled data.

In the present paper the hypothesis of seasonality of twin births in southeastern Brazil was investigated through the analysis of the variance of the trimestral and monthly twinning rate, as well as by testing whether the distribution of the trimestral twinning rate (total and dizygotic) fitted or not to a sinusoidal regression curve in which the observed periodicity corresponds to specific seasons.

\section{SUBJECTS AND METHODS}

A total of 154,699 deliveries from two important maternity hospitals in the State of São Paulo (86,804 in the Maternidade de Campinas and 67,895 in the Hospilal Santa Cata- 
rina, São Paulo) were investigated from 1984 to 1993 . They included 1,386 twin births ( 763 in the Maternidade de Campinas and 623 in the Hospital Santa Catarina). The twinning rate referred to the number of twin pair deliveries per 1,000 live and stillborn births children, fetuses with 500 g or less being excluded. Such fetuses were classified as abortions, since this weight corresponds to a gestation age between 20 and 22 weeks, as well as to $25 \mathrm{~cm}$ fetal length (2). As the twins have not been classified by any available zigosity test, the frequency of dizygotic pairs was estimated by means of the classical Weinberg's differential rule (22). Among the twins born in the Maternidade de Campinas it was possible to determine almost exactly the week of conception of 643 pairs.

The trimesters were considered as analogous to seasons, since in southeastern Brazil the first trimester corresponds practically to Summer, while Autumn, Winter and Spring are nearly coincident with the second, third and fourth trimester, respectively. The trimestral and monthly twinning rates in the maternity hospitals under study were compared by means of analysis of variance, and the search for curves that give best fit for the distribution of the data was performed by using the Curve Fits, Version 4.27 Program. Information on mean monthly temperature (maximum and minimum) and monthly rainfall (in $\mathrm{mm}$ ) from 1984 to 1993 in the city of Campinas, SP, Brazil was given by the Center of Teaching and Research in Agriculture of the State University of Campinas (UNICAMP).

\section{RESULTS AND DISCUSSION}

Table 1 shows the trimestral twinning rate (total and dizygotic) from 1984 to 1993 in the maternity hospitals under study. As it can be concluded from the analyses of variance in Table 2, the data obtained in these hospitals may be pooled together since no significant differences between them were observed. Moreover, Table 2 shows that neither significant trimestral differences nor interaction between hospitals and trimesters could be assigned for either total twinning rate as a whole or for dizygotic rate. The hypothesis of hospitals' identity and of absence of significant periodical differences in twinning rates or interaction has been confirmed even when the twinning rate was considered monthly (Tables 3 and 4).

In spite of the analyses in table 2 and 4 being sufficient for demonstrating absence of seasonality of twin birth in our data, another attempt was made for investigating an eventual periodicity of twinning rate. Thus, by analyzing the pooled trimestral data on Table 1 , it was observed that sinusoidal regression curves fit well to both the total twinning rate $(y=9.6254-1.3239 \sin ($ trimester +0.1035$)(r=0.33)$ and the dizygotic twinning rate $(y=4.8318-0.6970 \sin$ (trimester -0.0765$)(\mathrm{r}=0.35)$. Nevertheless, in both cases the periodicity observed did not correspond to specific seasons but to periods of about one and half years, as seen in Fig. 1 . The same picture emerged from the data based on the trimester of conception. Moreover, the Campinas data did not show significant correlations with temperature (maximum and minimum) or rainfall. These findings may suggest that previously reported "seasonality" might be just a coincidence, since the real cause of the observed periodicity remains unknown.

Acknowledgments: This study received support from CNPq, FAPESP and CAPES. 
Investigation on Seasonality of Twin Births in Brazil 399

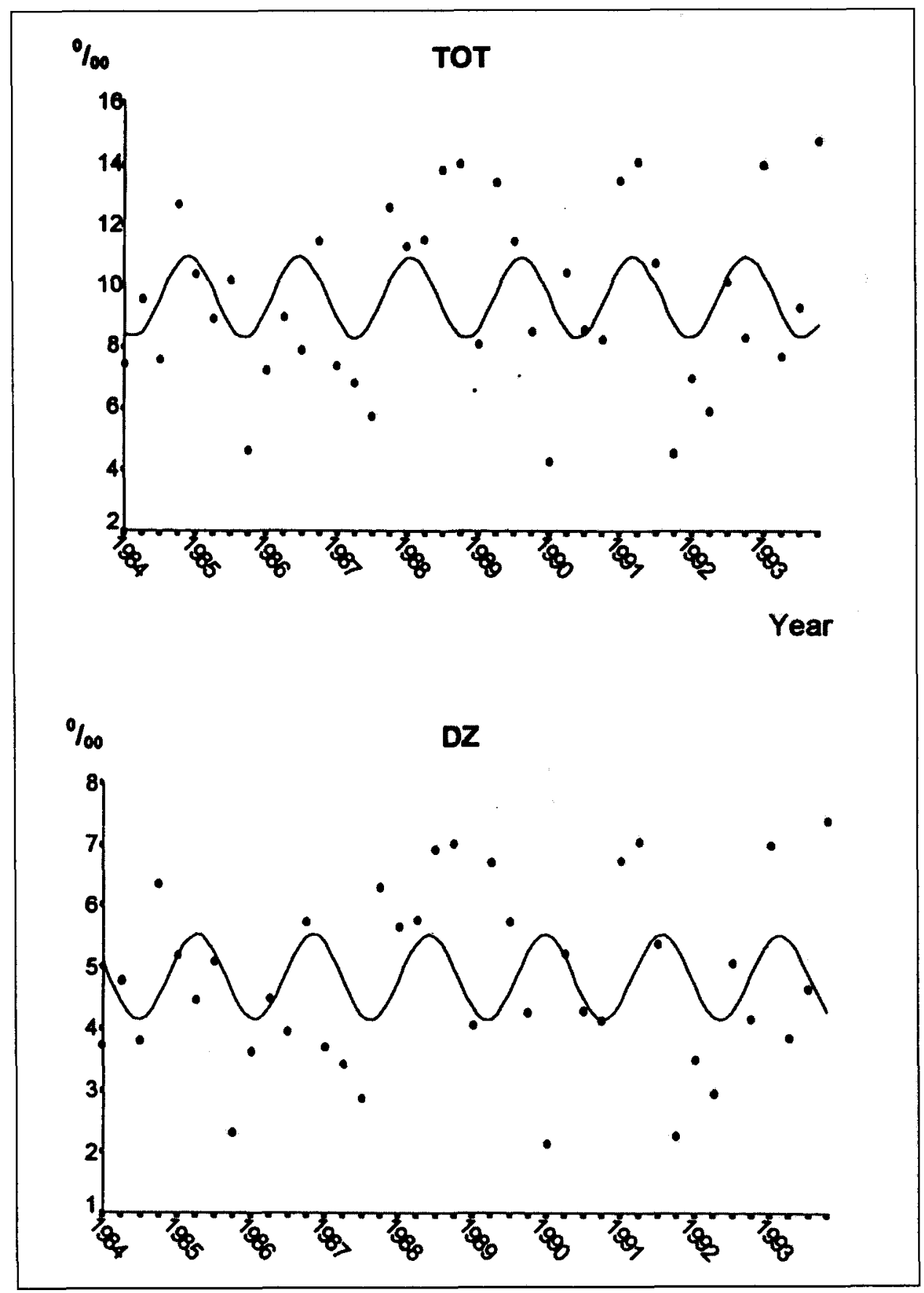

Fig. 1 - Sinusoidal regression curves for the trimestral distribution of the twinning rate as a whole (TOT) and of the dizygotic twinning rate (DZ). 
Table 1 - Trimestral twinning rate - total (TOT) and dyzigotic (DZ) - from 1984 to 1993 in two maternity hospitals at the State of Sao Paulo, Brazil (Maternidade de Campinas and Hospital Santa Catarina)

Maternidade de Campinas

\begin{tabular}{lrrrrrrrrr}
\hline & \multicolumn{2}{c}{ First } & \multicolumn{2}{c}{ Second } & \multicolumn{2}{c}{ Third } & \multicolumn{2}{c}{ Fourth } \\
Year & TOT & DZ & TOT & DZ & TOT & DZ & TOT & DZ \\
\hline 1984 & 9.69 & 5.28 & 5.96 & 3.41 & 10.54 & 5.75 & 8.35 & 5.57 \\
1985 & 8.94 & 4.47 & 8.40 & 4.20 & 8.64 & 5.18 & 7.69 & 2.99 \\
1986 & 9.74 & 3.34 & 9.79 & 7.04 & 8.05 & 1.79 & 9.63 & 7.34 \\
1987 & 4.51 & 2.58 & 8.66 & 4.07 & 9.48 & 3.79 & 9.57 & 5.74 \\
1988 & 9.77 & 6.51 & 6.66 & 4.44 & 9.19 & 7.88 & 11.46 & 5.73 \\
1989 & 6.56 & 3.50 & 8.33 & 7.50 & 9.57 & 3.83 & 7.35 & 1.96 \\
1990 & 11.55 & 0.96 & 7.66 & 4.31 & 8.01 & 4.74 & 5.08 & 3.05 \\
1991 & 10.40 & 3.12 & 12.82 & 5.70 & 9.20 & 4.09 & 9.30 & 5.86 \\
1992 & 7.87 & 4.63 & 9.36 & 3.74 & 13.83 & 1.98 & 8.72 & 3.67 \\
1993 & 8.17 & 7.27 & 6.84 & 2.56 & 8.27 & 3.48 & 8.28 & 4.60
\end{tabular}

Hospital Santa Catarina

\begin{tabular}{lrrrrrrrrr}
\hline & \multicolumn{2}{c}{ First } & \multicolumn{2}{c}{ Second } & \multicolumn{2}{c}{ Third } & \multicolumn{2}{c}{ Fourth } \\
Year & \multicolumn{1}{c}{ TOT } & DZ & TOT & DZ & TOT & DZ & TOT & DZ \\
\hline 1984 & 5.33 & 1.33 & 7.08 & 7.08 & 6.93 & 1.26 & 8.89 & 7.52 \\
1985 & 9.62 & 6.20 & 9.13 & 4.87 & 8.81 & 4.40 & 8.79 & 1.03 \\
1986 & 7.44 & 3.97 & 6.00 & 1.71 & 10.38 & 6.05 & 8.51 & 3.40 \\
1987 & 8.03 & 4.46 & 8.01 & 2.83 & 6.84 & 1.95 & 8.86 & 3.94 \\
1988 & 9.59 & 4.80 & 11.08 & 7.09 & 7.72 & 6.00 & 6.09 & 5.62 \\
1989 & 5.42 & 3.61 & 10.78 & 5.67 & 14.28 & 8.00 & 9.92 & 7.00 \\
1990 & 9.23 & 3.08 & 13.26 & 5.77 & 8.61 & 3.69 & 9.91 & 5.66 \\
1991 & 10.07 & 4.03 & 8.36 & 1.39 & 12.92 & 7.18 & 6.08 & 4.73 \\
1992 & 9.49 & 0.00 & 9.58 & 1.60 & 13.26 & 10.61 & 8.44 & 5.06 \\
1993 & 13.87 & 8.16 & 9.28 & 6.18 & 13.96 & 6.98 & 14.97 & 12.16
\end{tabular}

Both Hospitals

\begin{tabular}{lrrrrrrrr}
\hline & \multicolumn{2}{c}{ First } & \multicolumn{2}{c}{ Second } & \multicolumn{2}{c}{ Third } & \multicolumn{2}{c}{ Fourth } \\
Year & \multicolumn{1}{c}{ TOT } & DZ & TOT & DZ & TOT & DZ & TOT & DZ \\
\hline 1984 & 7.96 & 3.71 & 6.38 & 4.79 & 8.98 & 3.81 & 8.56 & 6.36 \\
1985 & 9.35 & 5.19 & 8.70 & 4.47 & 8.71 & 5.08 & 8,18 & 2.34 \\
1986 & 8.38 & 3.62 & 7.97 & 4.50 & 9.23 & 3.96 & 9,05 & 5.74 \\
1987 & 6.59 & 3.69 & 8.32 & 3.43 & 8.17 & 2.88 & 9,22 & 6.31 \\
1988 & 9.68 & 5.67 & 8.87 & 5.77 & 8.44 & 6.93 & 8.86 & 7.04 \\
1989 & 6.08 & 4.05 & 9.37 & 6.72 & 11.72 & 5.73 & 8.52 & 4.26 \\
1990 & 10.53 & 2.16 & 10.20 & 5.23 & 8.30 & 4.28 & 7.10 & 4.14 \\
1991 & 10.25 & 6.74 & 11.01 & 7.06 & 10.75 & 5.37 & 7.94 & 2.27 \\
1992 & 8.46 & 3.50 & 9.45 & 2.95 & 13.62 & 5.07 & 8.62 & 4.16 \\
1993 & 10.21 & 7.00 & 7.98 & 3.85 & 10.16 & 4.65 & 10.49 & 7.40 \\
\hline
\end{tabular}


Table 2 - Comparison of the trimestral data on Table 1 by analysis of variance

\begin{tabular}{|c|c|c|c|c|c|}
\hline \multirow{2}{*}{$\begin{array}{l}\text { Source of } \\
\text { variation }\end{array}$} & \multirow{2}{*}{$\begin{array}{l}\text { Degrees of } \\
\text { freedom }\end{array}$} & \multicolumn{2}{|c|}{ Total } & \multicolumn{2}{|c|}{ Dyzigotic } \\
\hline & & Sum of squares & $\mathrm{F}$ & Sum of squares & $\mathbf{F}$ \\
\hline Hospitals & 1 & 7.534 & 0.219 n.s. & 4.260 & 0.866 n.s. \\
\hline Seasons & 3 & 18.854 & 0.288 n.s. & 13.226 & 0.986 n.s. \\
\hline Interaction & 3 & 1.767 & 0.948 n.s. & 10.250 & 0.695 n.s. \\
\hline Error & 72 & 353.489 & & 354.154 & \\
\hline Total & 79 & 381.644 & & 381.890 & \\
\hline
\end{tabular}

Table 3 - Montlhy twinning rate from 1984 to 1993 in two maternity hospitals at the State of São Paulo, Brazil (Maternidade de Campinas and Hospital Santa Catarina)

Maternidade de Campinas

\begin{tabular}{lllllllllllll}
\hline Year & Jan & Feb & Mar & Apr & May & Jun & Jul & Aug & Sep & Oct & Nov & Dec \\
\hline 1984 & 1,09 & 0,94 & 0,88 & 0,94 & 0,39 & 0,41 & 0,87 & 1,41 & 0,87 & 0,69 & 0,58 & 1,21 \\
1985 & 1,26 & 0,55 & 0,88 & 0,81 & 1,00 & 0,72 & 0,51 & 0,81 & 1,28 & 0,62 & 0,78 & 0,92 \\
1986 & 0,99 & 1,35 & 0,47 & 0,89 & 1,06 & 1,00 & 1,05 & 0,57 & 0,77 & 1,02 & 1,41 & 0,44 \\
1987 & 0,69 & 0,46 & 0,19 & 1,02 & 0,87 & 0,75 & 1,08 & 0,87 & 0,88 & 0,70 & 0,78 & 1,37 \\
1988 & 0,66 & 1,15 & 1,16 & 0,70 & 0,78 & 0,52 & 0,81 & 0,81 & 1,11 & 1,53 & 0,70 & 1,18 \\
1989 & 0,49 & 0,55 & 0,93 & 0,78 & 0,71 & 1,01 & 0,54 & 0,83 & 1,59 & 1,18 & 0,68 & 0,32 \\
1990 & 1,39 & 0,81 & 1,21 & 1,25 & 0,56 & 0,46 & 0,53 & 1,44 & 0,45 & 0,32 & 0,90 & 0,30 \\
1991 & 1,19 & 0,84 & 1,08 & 1,36 & 1,58 & 0,88 & 0,45 & 1,43 & 0,90 & 1,13 & 0,78 & 0,86 \\
1992 & 0,67 & 0,86 & 0,84 & 1,02 & 0,41 & 1,38 & 0,78 & 1,37 & 1,93 & 0,56 & 1,52 & 0,54 \\
1993 & 0,82 & 0,70 & 0,92 & 0,67 & 0,38 & 1,00 & 0,69 & 0,88 & 0,91 & 0,70 & 0,55 & 1,23
\end{tabular}

Hospital Santa Catarina

\begin{tabular}{lllllllllllll}
\hline Year & Jan & Feb & Mar & Apr & May & Jun & Jul & Aug & Sep & Oct & Nov & Dec \\
\hline 1984 & 0,60 & $\mathbf{0 , 4 0}$ & $\mathbf{0 , 6 0}$ & 0,43 & 1,52 & 0,00 & 0,97 & 0,41 & 0,68 & 1,41 & 0,62 & 0,62 \\
1985 & $\mathbf{0 , 7 0}$ & $\mathbf{1}, 46$ & $\mathbf{0 , 8 9}$ & 0,40 & 1,33 & 0,93 & 1,03 & 0,67 & 0,94 & $\mathbf{0 , 8 4}$ & 0,97 & 0,83 \\
1986 & 0,95 & 0,93 & $\mathbf{0 , 4 1}$ & 0,26 & 0,91 & 0,63 & 1,59 & 0,91 & 0,64 & $\mathbf{0 , 9 6}$ & 0,97 & 0,63 \\
1987 & 1,04 & 0,56 & $\mathbf{0 , 8 0}$ & 0,67 & 1,00 & 0,74 & 0,15 & 0,79 & 1,09 & $\mathbf{0 , 6 0}$ & 0,75 & 1,28 \\
1988 & 0,91 & 0,94 & 1,02 & 1,21 & 1,35 & 0,78 & 0,79 & 0,65 & 0,87 & 0,41 & 1,18 & 0,27 \\
1989 & 0,36 & 0,59 & 0,67 & 1,62 & 1,00 & 0,66 & 1,55 & 1,55 & 1,18 & 0,81 & 1,30 & 0,89 \\
1990 & 0,53 & 0,59 & 1,63 & 1,00 & 1,02 & 2,01 & 1,22 & 0,92 & 0,39 & 0,64 & 1,10 & 1,22 \\
1991 & 0,42 & 1,02 & 1,52 & 1,16 & 0,61 & 0,70 & 1,08 & 1,34 & 1,46 & 0,57 & 0,62 & 0,64 \\
1992 & 0,74 & 0,91 & 1,19 & 0,72 & 1,14 & 1,02 & 1,64 & 0,75 & 1,64 & 1,48 & 0,79 & 0,25 \\
1993 & 0,99 & 0,81 & 2,22 & 0,97 & 0,94 & 1,09 & 0,80 & 2,30 & 1,05 & 1,32 & 0,90 & 2,23 \\
& & & & & & & & & & & (continued on page 402)
\end{tabular}


Both Hospitals

\begin{tabular}{lllllllllllll}
\hline Year & Jan & Feb & Mar & Apr & May & Jun & Jul & Aug & Sep & Oct & Nov & Dec \\
\hline 1984 & 0,89 & 0,73 & 0,77 & 0,76 & 0,85 & 0,26 & 0,91 & 1,01 & 0,78 & 0,99 & 0,60 & $\mathbf{0 , 9 8}$ \\
1985 & 1,01 & 0,91 & 0,89 & 0,64 & 1,14 & 0,80 & 0,73 & 0,75 & 1,13 & 0,72 & 0,86 & 0,88 \\
1986 & 0,97 & 1,15 & 0,44 & 0,60 & 0,99 & 0,83 & 1,32 & 0,75 & 0,71 & 0,99 & 1,19 & 0,54 \\
1987 & 0,89 & 0,52 & 0,54 & 0,81 & 0.93 & 0,74 & 0,64 & 0,83 & 0,99 & 0,65 & 0,76 & 1,33 \\
1988 & 0,77 & 1,04 & 1,09 & 0,96 & 1,06 & 0,65 & 0,80 & 0,73 & 0,99 & 0,99 & 0,93 & 0,74 \\
1989 & 0,44 & 0,57 & 0,81 & 1,13 & 0,83 & 0,86 & 0,99 & 1,15 & 1,39 & 1,00 & 0,94 & 0,59 \\
1990 & 1,01 & 0,71 & 1,39 & 1,14 & 0,77 & 1,17 & 0,83 & 1,21 & 0,43 & 0,46 & 0,98 & 0,69 \\
1991 & 0,84 & 0,92 & 1,27 & 1,27 & 1,20 & 0,81 & 0,71 & 1,40 & 1,14 & 0,89 & 0,71 & 0,77 \\
1992 & 0,69 & 0,88 & 0,97 & 0,90 & 0,69 & 1,25 & 1,09 & 1,14 & 1,83 & 0,89 & 1,27 & 0,44 \\
1993 & 0,88 & 0,74 & 1,40 & 0,78 & 0,57 & 1,03 & 0,73 & 1,34 & 0,95 & 0,91 & 0,66 & 1,56 \\
\hline
\end{tabular}

Table 4 - Comparison of the monthly data on Table 3 by analysis of variance

\begin{tabular}{lcrc}
\hline $\begin{array}{l}\text { Source of } \\
\text { variation }\end{array}$ & $\begin{array}{c}\text { Degrees of } \\
\text { freedom }\end{array}$ & $\begin{array}{c}\text { Sum of } \\
\text { squares }\end{array}$ & F \\
\hline Hospitals & 1 & 21.312 & 1.476 n.s. \\
Months & 11 & 120.416 & 0.758 n.s. \\
Interaction & 11 & 150.047 & 0.945 n.s. \\
Error & 216 & $3,119.114$ & \\
Total & 239 & $3,410.889$ & \\
\hline
\end{tabular}

\section{REFERENCES}

1. Beiguelman B, Villarroel-Herrera HO (1992): Seasonal variation in twin birth rates in Brazil. Rev Brasil Genet 15: 703-706.

2. Belitzky R, Fescina R, Ucieda F (1978): Definiciones y terminologia aplicables al periodo perinatal. Recomendaciones de la Organización Mundial de la Salud y Modificaciones de la FIGO. Publicación Científica del CLAP No. 757: 136-147.

3. Bonnelykke B, Sogaard J, Nielsen J (1987): Seasonality in twin birth rates, Denmark, 1936-84. J Epidemiol Community 41: 338-343.

4. Czeizel A (1974): Unexplainable demographic phenomena of multiple births in Hungary. Acta Genet Med Gemellol 22 (Suppl.): 214-218.

5. Edwards J (1938): Season and rate of conception. Nature 142: 357 .

6. Elwood JM (1978): Maternal and environmental factors affecting twin births in Canadian cities. Br J Obstet Gynecol 85: 351-358, 1978.

7. Erhard CL, Nelson FG, Pakter J (1971): Seasonal patterns of conception in New York City. Am J Publ Health 61: 2246-2258.

8. Gedda L (1994): Twin study today. Acta Genet Med Gemellol 43: 3-15, 1994. 
9. Gedda L, Brenci G, Carrega R (1989): Seasonality in twins vs. single births. Abstracts, Sixth Int. Cong Twin Studies, Acta Genet Med Gemellol 38 (1-2): 131.

10. Imaizumi Y (1992): Twinning rates in Japan 1951-1990. Acta Genet Med Gemellol 41: 165175.

11. Imaizumi Y, Asaka A, Inouye E (1980): Analysis of multiple birth rates in Japan. V. Seasonal and social class variations in twin births. Jap J Human Genet 25: 299-307.

12. James WH (1976) Seasonality and twin births. Ann Hum Biol 3: 193-195.

13. James WH (1980): Seasonality in twin and triplet births. Ann Hum Biol 7: 163-175.

14. Kamimura K (1976): Epidemiology of twin births from a climatic point of view. Br J Prev Soc Med 30: 175-179.

15. Nonaka K, Miura T (1987): Incidence of twinning in London from 1581 to 1760 . Acta Genet Med Gemellol 36: 365-372

16. Nonaka K, Miura T, Peter K (1989): Seasonal effects on twinning in a fertile population, the Hutterites. Abstracts, Sixth Int. Cong. Twin Studies, Acta Genet Med Gemellol 38 (1-2): I 12 113.

17. Nylander PPS (1981): The factors that influence twinning rates. Acta Genet Med Gemellol 30: 189-202.

18. Picard R, Fraser D, Hagay Z J, Leiberman J R (1990): Twinning in Israel. Seasonal variation effects of ethnicity, maternal age and parity. J Reprod Med 35: 163-167.

19. Richter J, Miura T, Nakamura I, Nonaka K (1984): Twinning rates and seasonal changes in Görlitz, Germany, from 1611 to 1860. Acta Genet Med Gemellol 33: 121-124.

20. Selvin S, Janerich DT (1972): Seasonal variation in twin births. Nature 237: 289-290.

21. Timonen S, Carper E (1968): Multiple pregnancies and photoperiodicity. Ann Chir Gynaecol Fenn 57: 135-138.

22. Weinberg W (1901): Beiträge zur Physiologie und Pathologie der Mehrlingsgeburten beim Menschen. Pflügers Arch. Ges. Physiol. 88: 346-430.

23. Zahalkova Mi (1974): Multiple births in Southern Moravia. Acta Genet Med Gemellol 22 (Suppl.): 210-213.

Correspondence: Prof. Dr. Bernardo Beiguelman, Departamento de Genética Médica, Faculdade de Ciências Médicas, C.P. 6111, UNICAMP, 13081-970 Campinas, SP, Brazil. 